\title{
LOS SEÑORES DE LA ENTIDAD POLÍTICA DE 'IK'
}

\section{ERIK Velásquez García}

\begin{abstract}
Resumen: Este artículo constituye el primer intento de reconstrucción de la dinastía real de 'Ik' que se ha publicado. En 1973, el glifo emblema de la entidad política de 'Ik' fue asociado por Joyce Marcus con el sitio arqueológico de Motul de San José, asentado en las cercanías del lago Chaltunhá o Petén Itzá. Desde entonces, diversos datos arqueológicos y epigráficos han fortalecido la hipótesis de que la entidad política de 'Ik' tuvo su sede en esa zona del Petén, si bien su primera capital conocida residió posiblemente en Bejucal. Del mismo modo, se contempla la posibilidad de que más de un sólo dignatario haya portado simultáneamente el glifo emblema de ' $/ k$ ', pues fue un título regional usado por distintas ciudades del mismo estado. En este trabajo se identifican dos gobernantes de 'Ik' del Clásico temprano, siete u ocho del Clásico tardío y uno o dos del Clásico terminal, aunque en las vasijas producidas en ocho dé Clásico tartio y una o dos del Clásico ten in án la region existen oros dignatarios cuyos nomber de fechas en Rieda de Calendaio. Una aportion significativa del texto reside en identificar los sitios arqueológicos del Petén, Petexbatún
\end{abstract}

Palabras clave: entidad política, glifo emblema de 'Ik', máscaras de "rayos X", Motul de San José, vasijas mayas

Abstract: This article is the first attempt to reconstruct the 'Ik' dynasty that has been published. At 1973, the emblem glyph of 'lk' politic was associated by Joyce Marcus with the archaeological site of Motul San Jose, seated near Chaltunhá or Peten Itza lake. Since then, various archaeologisite of Motul San Jose, seated near Chaltunha or Peten Itza lake. Since then, various archaeologi-
cal and epigraphic data have been suported the hypothesis that ' $I \mathrm{k}$ ' politic had its seat at the Peten area, although his first known capital was possibly located at Bejucal. At the same way, we Peten area, although his first known capital was possibly located at Bejucal. At the same way, we
see the posibility that more than one dignitary had ported simultaneously the emblem glyph of see the posibility that more than one dignitary had ported simultaneously the emblem glyph of
'lk', because it was a regional title used by different cities of the same state. This research identified two 'Ik' rulers of the Early Classic, seven or eight of the Late Classic, and one or two of the Last Classic, although in the vessels produced in the region there are other dignitaries' names which are difficult to understand or without dates of Calendar Round. A significant contribution of the text consist in identifying the archeological sites in Peten, Petexbatún and Usumacinta with which the lordship of 'lk' had very diverse relationsips.

Key

Recepción: 14 de enero de 2008

Aceptación: 20 de octubre de 2008 



\title{
LOS SEÑORES DE LA ENTIDAD POLÍTICA DE 'IK'*
}

\author{
ERIK VelásQuez García
}

Instituto de Investigaciones Estéticas, unam

\begin{abstract}
A finales de la década de 1970, Michael D. Coe (1978: 130-134) destacó la existencia de un grupo de vasos mayas sin procedencia arqueológica conocida, pero asociados entre sí por un puñado de atributos formales: engobe crema con bordes pintados de negro y glifos de color rosa, así como escenas dramatizadas con ejecutantes obesos vestidos con atuendos fantásticos, que incluían máscaras pintadas en corte transversal o perspectiva de "rayos X" (Velásquez García, 2007), no sin ponderar su excelente calidad de dibujo, ejecutado con una línea fluida y delicada. Coe propuso que el locus de este estilo pudo haber residido en Motul de San José, puesto que los vasos incluían el glifo emblema de 'Ik', ${ }^{1}$ asociado por Joyce Marcus (1973: 912; 1976: 17-19) con ese sitio del Petén.

Motul de San José se ubica $3 \mathrm{~km}$ al norte del lago Chaltunhá o Petén Itzá (figura 1). Fue descubierto en 1895 por Teobert Maler (1910: 132-135), cuya descripción constituyó por muchos años el único reporte académico confiable, hasta que Sylvanus G. Morley (1937: 415-421) visitó el sitio en mayo de 1915 y publicó un breve estudio sobre sus estelas. Desde entonces, y quizá con la excepción de José Antonio Villacorta (1928), nadie se había vuelto a interesar seriamente por el sitio, pero en 1998 un grupo de investigadores coordinado por Antonia E. Foias (1999; 2003a; 2003b; Emery, 2003; Halperin, 2004; 2006; Kennedy Thornton, 2008; Moriarty, 2004a; 2004b), emprendió un proyecto arqueológico que incluía trabajos de reconocimiento en la zona, mapeo y excavación de sondeo.

*N. del E. Para las palabras en maya se respetó la ortografía del autor.

${ }^{1}$ En este artículo emplearé como herramienta de trabajo las reglas propuestas por Alfonso Lacadena y Søren Wichmann (2004; s.f.) para la transliteración y transcripción de jeroglíficos mayas, sì bien debo aclarar que estoy consciente de que la longitud vocálica en las inscripciones clásicas es uno de los debates vigentes de la epigrafía (Robertson et. al., 2007). En la representación de palabras de origen maya usaré las siguientes grafías: a, e, i, o, u, aa, ee, ii, oo, uu, a' $\mathrm{a}^{\prime} / \mathrm{a}^{\prime}(\mathrm{a}), \mathrm{e}^{\prime} / \mathrm{e}^{\prime}(\mathrm{e}), \mathrm{i}^{\prime} / \mathrm{i}^{\prime}(\mathrm{i}), \mathrm{o}^{\prime} / \mathrm{o}^{\prime}(\mathrm{o})$ $u^{\prime} / u^{\prime}(u), b^{\prime}, c^{\prime}, c^{\prime}, h, j, k, k^{\prime}, l, m, n, p, s, t, t^{\prime}, t z, t z ', w, x, y$, , pero en los nombres de los idiomas $\mathrm{u}^{\prime} / \mathrm{u}^{\prime}(\mathrm{u}), \mathrm{b}, \mathrm{ch}, \mathrm{ch}, \mathrm{h}, \mathrm{j}, \mathrm{k}, \mathrm{k}, \mathrm{l}, \mathrm{m}, \mathrm{n}, \mathrm{p}, \mathrm{s}, \mathrm{t}, \mathrm{t}^{\prime}, \mathrm{tz}, \mathrm{tz}, \mathrm{w}, \mathrm{x}, \mathrm{y},{ }^{\prime}$, pero en los nombres de los idiomas
mayances, así como en la traducción de los términos calendaricos, procuraré apegarme al uso tradimayances, así como en la traducción de los términos calendáricos, procuraré apegarme al uso tradi-
cional, basado en la ortografía del siglo xvi (por ejemplo chol, yucateco, Ik, Akbal, Kan, Chicchan, etc. en lugar de ch'ol, yukateko, 'Ik', 'Áak'b’al, K'an, Chikchan, etc.) cuyos primeros intentos de adaptación al alfabeto romano se deben probablemente a fray Luis de Villalpando (?-1552). Para designar signos no descifrados o de lectura dudosa me valdré del célebre catálogo de jeroglíficos mayas de J. Eric S. Thompson (1962), mientras que la mayor parte de las vasijas serán identificadas por su número dentro del catálogo de Justin Kerr (1989; 1990; 1992; 1994; 1997; 2000). Cabe la pena mencionar que en este texto utilizaré la nomenclatura de las estelas y la designación alfanumérica de los cartuchos glíficos que propuso Sylvanus G. Morley (1937: 417-421).
\end{abstract}

VELASQUEZ / LOS SEÑoRES DE LA ENTIDAD POLÍtica DE 'IK' 


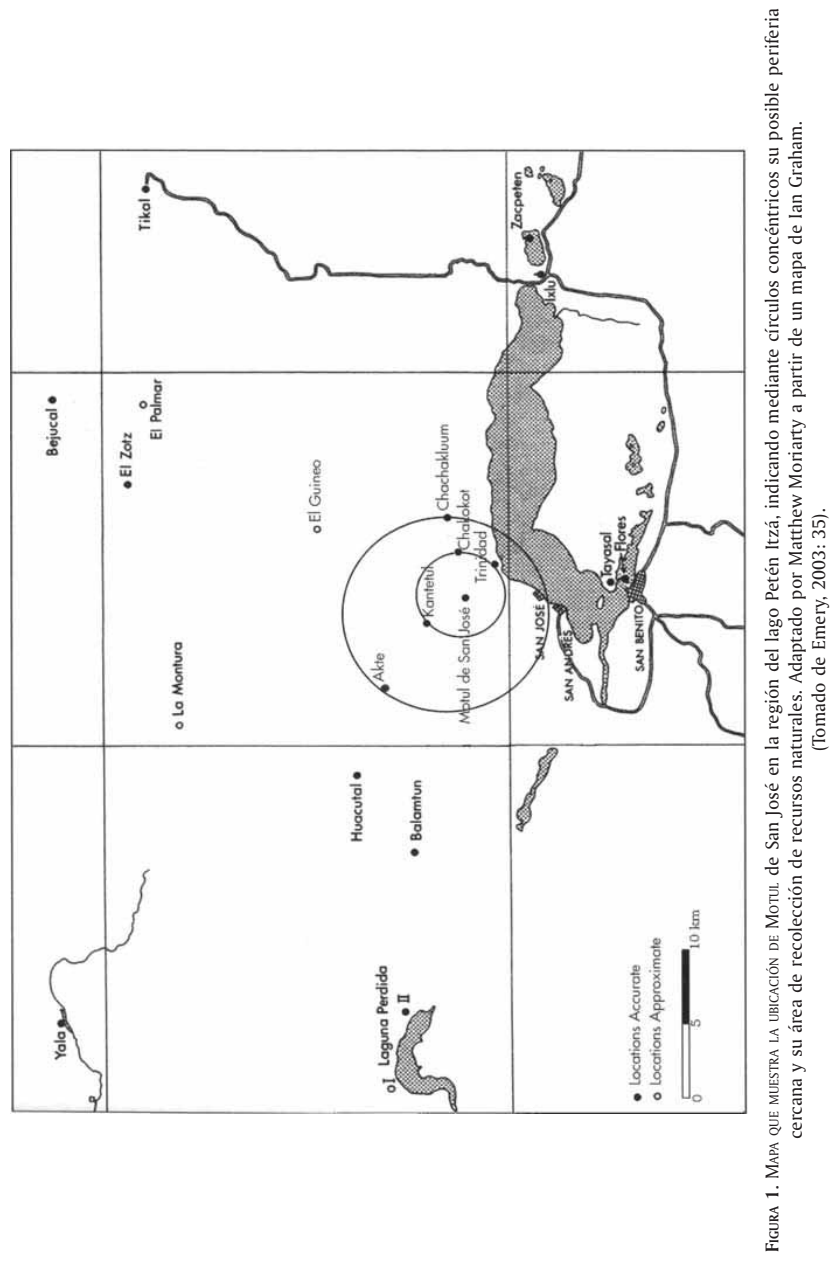


Como resultado de ello contamos con un excelente plano del área central del sitio, que incluye 144 estructuras agrupadas en cinco complejos arquitectónicos, cuya nomenclatura va de la $\mathrm{A}$ a la $\mathrm{E}$. El área medular se encuentra alrededor de la Plaza Principal, donde se ubican cinco de las seis estelas conocidas, mientras que la Acrópolis cierra el conjunto por el norte y parece haber formado el principal palacio real de 'Ik' (Foias, 2003a: 21, 23, 27; 2003b: 4; Moriarty, 2004b: 31).

El Proyecto Arqueológico Motul de San José ha podido determinar que el sitio tuvo una larga ocupación que abarca desde 600 a. C. hasta 1250 d.C., aunque durante el Clásico Temprano fue casi abandonado. La mayor parte de las estructuras pertenecen al Clásico Tardío, periodo donde experimentó su más alta concentración demográfica. David S. Stuart y Stephen D. Houston (1994: 27-28) han sugerido que el nombre clásico de la ciudad parece haber sido 'Ik'a', ${ }^{2}$ como se desprende de un pasaje contenido en la Estela 2 (C5), que consigna un rito ejecutado en 'Ik'a' ('u-ti-ya-'IK'-'a, 'u[h]tiiy 'Ik'a['], 'ocurrió en 'Ik'a'). El topónimo de 'Ik'a' se encuentra atestiguado en monumentos y vasos que mencionan a individuos de Motul de San José. ${ }^{3}$

Durante el siglo vill los soberanos de 'Ik' no parecen haber centralizado el poder político en Motul, sino que esta ciudad era parte de una red de sitios secundarios y terciarios que levantaban sus propios monumentos y poseían sus propios talleres de cerámica. Mientras que Motul de San José pudo haber sido el sitio principal, Trinidad de Nosotros parece haber funcionado como puerto de la entidad política de 'Ik' en el lago Chaltunhá (Moriarty, 2003; Moriarty y Foias, 2007), en tanto que los señores de Akte y Chachakluum pudieron también utilizar el emblema de 'Ik'. ${ }^{4}$ Stuart y Houston (citados en Foias, 1999: 947; 2003a:19, nota 6) sugieren que durante esta época varios sitios pudieron operar como capitales múltiples del reino, mientras que Nikolai Grube y Simon Martin

${ }^{2}$ La etimología de 'lk'a' es incierta todavía. David S. Stuart y Stephen D. Houston (1994: 28) supusieron que el elemento final del locativo 'Ik'a' probablemente conllevaba el significado de $h a$ ', 'agua', lo que remitiría a una pareja antitética 'ik'-ha', 'viento-agua' (véase Hull, 2003: 465). Otra alternativa fue propuesta por Raphael Tunesi y Luís Lopes (2004: 3), quienes consideraron la posibilidad de que el sufijo $-a$ ', en el caso del locativo ' 'k'a', pueda ser simplemente una partícula para nombres de lugar: en cuyo caso 'Ik'a' podría traducirse como "Lugar Ventoso".

3 Por ejemplo la Estela 21 de Yaxchilán (pH8) y el vaso K2295.

${ }_{4}^{4}$ El único monumento que se preserva en Akte es la Estela 1, de 743 o 747 d.C., pero ningún glifo emblema puede ser identificado en él, a causa de su erosión tan avanzada (ver Mayer, 2000). No se de alguna escultura preservada en Chachakluum; no obstante, Matthew D. Moriarty (2004b: 36 opina que este sitio era un asentamiento secundario de la entidad política de ' $k$ ', mientras que Akte pudo haber operado como centro administrativo o residencia rural de los mismos señores de 'lk'. E análisis de activación neutrónica, efectuado por Ronald L. Bishop, sugiere que había cuatro grupos químicos de cerámica manufacturados en Motul de San José, más un quinto grupo de composición disimilar, que probablemente procede de los sitios satélites del área (Reents-Budet et al., 2007: 1142). La existencia de vasijas (K1463, K1399, K2795 y K3054) donde aparecen simultáneamente dos señores divinos de 'Ik' sugiere que más de una persona podía llevar ese glifo emblema. David S. Stuart (comunicación personal, 25 de noviembre de 2007) incluso sospecha que debieron existir varios asentamientos en la región del lago Petén Itzá, cuyos señores detentaban ese glifo emblema.

VELASQUEZ / LOS SEÑORES DE LA ENTIDAD POLÍTICA DE 'IK' 
(2000: 92) consideran que el glifo emblema de 'Ik' era un título regional que fue utilizado en distintas ciudades del mismo estado al mismo tiempo. Estas aseveraciones parecen confirmarse por el hecho de que se han identificado diversos estilos de cerámica que portan el emblema de 'lk' (véase Reents-Budet et al. 1994: 172-179). Esto último, aunado a la diversidad química en la composición de sus pastas, analizadas por Ronald L. Bishop (Reents-Budet et al., 2007: 1142), confirma la sospecha de que el señorío de 'Ik' estuvo constituido, durante el Clásico Tardío, por un conjunto de sitios cuyos señores participaban del poder político del reino. No obstante, conviene decir que de los cinco grupos químicos principales, cuatro han sido asignados a Motul de San José (véase nota 4), por lo que quizá los mejores, y la mayor parte de los vasos, se producían directamente para la corte del señor divino que habitaba en la Acrópolis de 'Ik'a', sitio que puede ser considerado como el epicentro del estilo y sus talleres de cerámica (Looper, Reents-Budet y Bishop, 2009: 132).

\section{Fuentes para el estudio de la historia dinástica de 'lk'}

A diferencia de muchos otros sitios mayas que cuentan con abundantes monumentos, en Motul de San José sólo existen seis estelas rotas y mal preservadas, por lo que no es posible de momento contar con una secuencia precisa de sus gobernantes. Las pocas referencias escritas que existen sobre el señorío de 'Ik' proceden de textos epigráficos foráneos y de algunos datos que han podido rescatarse de vasijas que cuentan con registros históricos. Debemos advertir, sin embargo, que la determinación de las fechas pintadas en los vasos no siempre es segura, puesto que sus superficies están desgastadas y por ende, la identificación de algunos glifos se presta a discusión. La historia de los señores de 'Ik' es por ahora una sucesión de lagunas y episodios aislados.

Yune' B'ahlam de Bejucal

La referencia conocida más temprana a un gobernante de 'Ik' procede de Bejucal, que se encuentra más de $30 \mathrm{~km}$ al noreste de Motul de San José. La Estela 2 (figura 2) de ese sitio (véase Schele y Grube, 1994: 88) 'fue hincada en el suelo' (tz'alh/paj) con motivo del final de periodo de 393 d.C. (8.17.17.0.0). El agente de esta ceremonia parece llamarse Yune' B'ahlam (["yu]-ne-B'ALAM) (Stuart, 2000: 479) y porta el emblema de los señores de 'Ik'. Un número distancia erosionado de al menos doce años remite a una fecha de 381 d. C., que corresponde probablemente a la entronización del soberano; éste se autonombra como '[vasallo] de' [...] K'ahk', kalo'mte', 'ajaw. Algunos epigrafistas (Schele y Grube, 1994: 88; Grube y Martin, 2000: 92-93; Stuart, 2000: 479; Martin y Grube, 1994: 10, nota 5; 2008: 30) sospechan que este nombre corresponde al de Sihyaj K'ahk', lugarteniente 


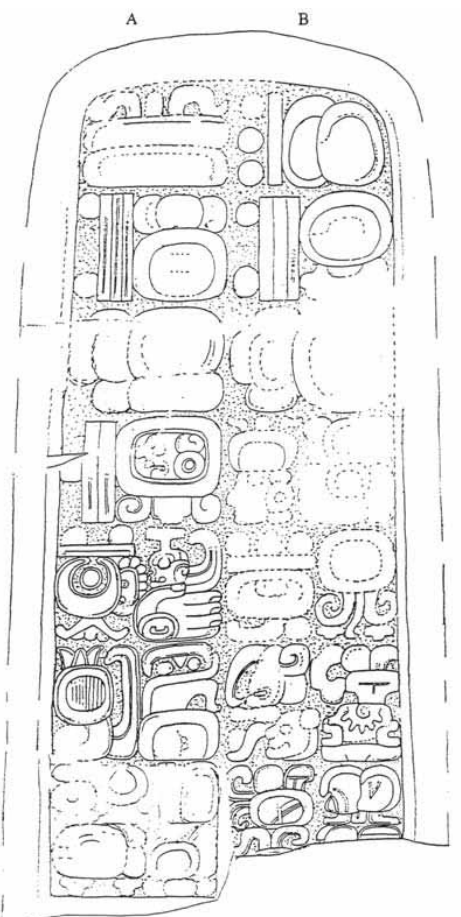

Figura 2. Estela 2 de Bejucal que atestigua la existencia del gobernante Yune' B'ahlam (C6b-D6b): ["yu]-ne-B'ALAM K'UH-'IK'-'AJAW 'AK?, [Yulne' B'ahlam, K'uh/ul] 'lk' 'Ajaw, 'Ahk(?) (ca. 381-393 d.C.) Boceto de Linda Schele.

de un posible señor teotihuacano que instaló gobernantes en algunos sitios del Petén a finales del siglo iv (ver Stuart, 2000; Martin y Grube, 2008: 29-31).

Como puede advertirse en ciertos monumentos de Tikal, ${ }^{5}$ Sihyaj K'ahk' portaba el título supremo de kalo'mte', que denotaba la máxima autoridad política a la que podía aspirar un hombre maya, pues servía para designar a los seño-

${ }^{5}$ Especialmente la Estela 31 (C22-D22) y el llamado Marcador (A8-B8). 
res que sujetaron territorios más allá de sus propios dominios (Harrison, 1999: 79; Stuart, 2000: 486-487; Houston y Stuart, 2001:60; Martin, 2001:106; Boot, 2005:511). Aunque no se sabe que Sihyaj K'ahk' haya gobernado alguna ciudad maya, su función como probable agente de la ascensión de Yune’ B’ahlam sugiere que durante esta época Bejucal pertenecía a la misma esfera política que Tikal (ver Schele y Grube, 1994: 88; Martin y Grube, 1995: 44). En un grabado mural no publicado, dispuesto cerca de la ciudad, también se encuentra escrito un emblema de 'Ik' (Reents-Budet et al., 1994: 226, nota 31). Este dato, y el hecho de que Motul de San José estuvo casi despoblado durante el Clásico Temprano (Foias, 2003b: 2; Moriarty, 2004b: 38), refuerzan la sospecha de que el señorío de 'Ik' tuvo su sede temprana en Bejucal.

\section{Sak 'Uhx 'Ook K'inich}

La Fórmula Dedicatoria del vaso inciso K3844 sugiere que perteneció a una mujer del reino de Hix Witz. ${ }^{6}$ Su escena contiene una procesión de ocho personajes que se dirigen hacia un santuario provisto de techumbre acortinada y emplumada, donde se quema incienso en honor de un bulto personificado que reposa sobre una banqueta y está cubierto con piel de jaguar. Como ha notado Luis Lopes (2002), el segundo personaje de la procesión que se perfila hacia la derecha es un gobernante de 'Ik'; su tocado remata en el llamado dios bufón (Sak Hu'nal), símbolo de la realeza maya, y la glosa escrita sobre él lo nombra como Sak 'Uhx 'Ook K'inich, [señor] divino de 'Ik' (SAK-3-'OK K'INICH-chi K'UH-'IK') (figura 2). ${ }^{7}$ Ninguna fecha aclara en qué periodo vivió ese mandatario, pero el estilo gráfico y dibujístico de este vaso parece relativamente temprano.

\footnotetext{
${ }^{6}$ Como fue sugerido primero por Stuart, la entidad política de Hix Witz parece haber estado conformada por distintos asentamientos ubicados en la ribera sur del río San Pedro Mártir, entre los que se encuentran La Joyanca, El Pajaral y Zapote Bobal (véase Breuil-Martínez et al., 2004).

${ }^{7}$ El logograma de 'IK' (T503) que aparece en esta cláusula nominal no se encuentra acompañado por el de 'AJAW (T168) y, en cambio, está superfijado por el signo T285, de posible valor fonético hu. Erik Boot (correspondencia enviada el 20 de julio de 2007) ha sugerido que la composición T285:503 podría leerse hux (hu-HUX?), 'aliento' o 'vapor', por lo que el personaje que aparece en el vaso K3844 quizá sea una deidad asociada con alguna entidad anímica (K'uh Hux, 'dios del aliento'). No obstante, su tocado de dios bufón (Sak Hu'nal) no permite descartar la posibilidad de que se trate de algún gobernante de 'Ik', aunque en apariencia carezca del logograma 'AJAW (T168), que suele acompaña a los glifos emblema. Otra posibilidad es que la composición T285:503 sea tan sólo un dígrafo del logograma 'IK' (Nikolai Grube, comunicación personal, 15 de noviembre de 2007). Cabe la pena destacar que Sak 'Uhx 'Ook operaba también como el nombre de un wahyis asociado con la región de Río Azul (Grube y Nahm, 1994: 697; Looper, Reents-Budet y Bishop, 2009: 135).
} 
El vasallo de Jasaw Chan K'awiil I

Una laguna de 308 años separa la Estela 2 de Bejucal de la siguiente mención fechada sobre un señor de 'Ik'. Ésta se encuentra en la Estela 1 (figura 3) de Motul de San José (ver Schele y Grube, 1994: 145), que consigna la entronización de un soberano en 701 d.C. (9.13.9.1.17), cuyo nombre se encuentra erosionado, pero el texto de la estela finaliza con una mención de Jasaw Chan K'awiil I, señor de Tikal (682-734 d.C.). Aunque la frase que conecta el nombre de los dos mandatarios está desgastada, ${ }^{8}$ algunos autores (Schele y Grube, 1994: 145; Martin y Grube, 1995: 44; 2008: 45-46; Foias, 2003a: 19; 2003b: 2; Moriarty, 2004b: 31) consideran que el soberano de Tikal se encuentra supervisando la ascensión del de Motul. ${ }^{9}$ Basados en sus propias inferencias, Dorie J. Reents-Budet y Ronald L.

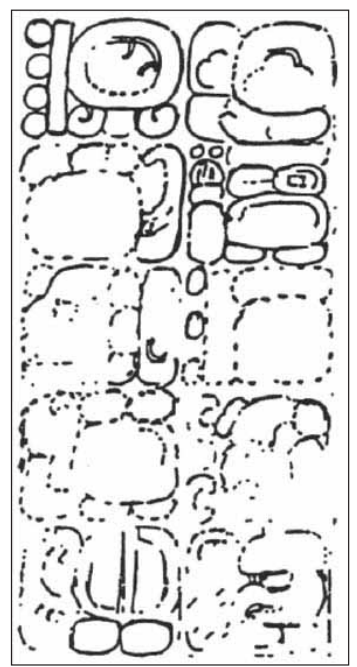

Figura 3. Fragmento de la Estela 1 de Motul de San José (A8-B12), ubicada al oeste de la Plaza Principal. Menciona la ascensión de un gobernante del linaje de 'lk' en 701 d. C., bajo los auspicios de Jasaw Chan K'awiil I de Tikal. Boceto de Linda Schele.

(Tomado de Schele y Grube, 1994: 145).

${ }^{8}$ Nikolai Grube afirma que se trata de 'es el vasallo de' (ya-'AjAW, yajaw).

${ }^{9}$ La estrecha relación que existió entre las entidades políticas de 'lk' (Motul de San José) y Mutu'l (Tikal) pudo quedar plasmada de algún modo en el nombre de Motul de San José, que no sólo recuerda al señorío de Mutu'l, sino al antiguo topónimo de Tikal: Yax Mutu’l (Martin y Grube, 2008: 30).

VELASQUEZ / LOS SEÑORES DE LA ENTIDAD POLÍtICA DE 'IK' 
Bishop (2003: 27; Reents-Budet et al., 2007: 1144) también sugieren que entre 700 y 726 gobernó en la entidad política de 'Ik' un soberano llamado Sak Muwaan (figura 3). De tener razón, el nombre de ese mandatario pudiera haber sido el sujeto de la entronización mencionada en la Estela 1 de Motul de San José (A10), aunque es preciso advertir que no he podido identificar de forma clara el nombre de Sak Muwaan en las imágenes existentes de ese monumento. ${ }^{10}$

\section{Sak Muwaan}

Los datos biográficos con que contamos de Sak Muwaan son escasos y episódicos. Él aparece pintado en el vaso K2803 cuando aún era joven, y participó en un juego de pelota contra el señor de Hix Witz (ver Tokovinine, 2002: 5; Zender, 2004: 11). Aunque el texto del vaso aclara que era 'señor divino de 'Ik', probablemente se trata de una mención anacrónica, dado que también se le llama chak ch'ok kele'm, 'gran fuerte joven', apelativos que normalmente son portados por los herederos de la más alta nobleza (ver Houston y Stuart, 1998: 79; 2001: 67, 72; MacAnany y Plank, 2001: 113; Le Fort, 2003: 90; Jackson, 2005: 211, 217, 227). La pertenencia de este vaso al corpus de cerámica 'Ik' no está comprobada, y su Fórmula Dedicatoria sugiere que perteneció a un señor de la entidad política de Hix Witz (Miller y Martin, 2004:91). Marc U. Zender (2004:11) opina que el vaso fue comisionado por el gobernante de Hix Witz y obsequiado al de 'Ik'.

Otra vasija interesante es la K2784. Su Fórmula Dedicatoria aclara que perteneció a K'eey ti Chan, "jugador de pelota sabio", quien era hijo de Sak Muwaan. La imagen representada en el vaso tuvo lugar durante la entronización de Tahn Tuun Chaahk como gobernante de Maan, señorío asociado con el moderno sitio de La Florida, ${ }^{11}$ y el análisis químico efectuado por Bishop confirma que fue elaborado con arcillas de esa región. Ello sugiere que el hijo del señor de 'Ik' viajó hasta La Florida, en la ribera del San Pedro Mártir, para presenciar el ascenso de su soberano; ahí mismo pudo haber recibido ese vaso como regalo, aunque aparentemente nunca ascendió al trono (Reents-Budet y Bishop, 2003: 27; Reents-Budet et al., 2007: 1144). Cabe aclarar que en el vaso Sak Muwaan porta los títulos de yax ti' ('primera boca'), kalo'mte' y b'aah kab' ('cabeza' o 'príncipe de la tierra'), lo que sugiere que mantenía cierta autonomía y poder regional, aun en el caso de que fuera vasallo de Tikal.

${ }^{10}$ La Estela 1 de Motul de San José ya sólo se puede admirar en las fotografías de Teobert Maler (1895), mismas que no están publicadas, sino resguardadas en el archivo del proyecto Corpus of Maya Hieroglyphic Inscriptions (смнI) de la Universidad de Harvard.

${ }^{11}$ De acuerdo con Reents-Budet (Reents-Budet y Bishop, 2003: 27; Reents-Budet et al., 2007: 1144), Stanley P. Guenter identificó el glifo emblema de Maan o Namaan con La Florida, otro sitio ubicado en la ribera del San Pedro Mártir, aunque más occidental que el señorío de Hix Witz. 

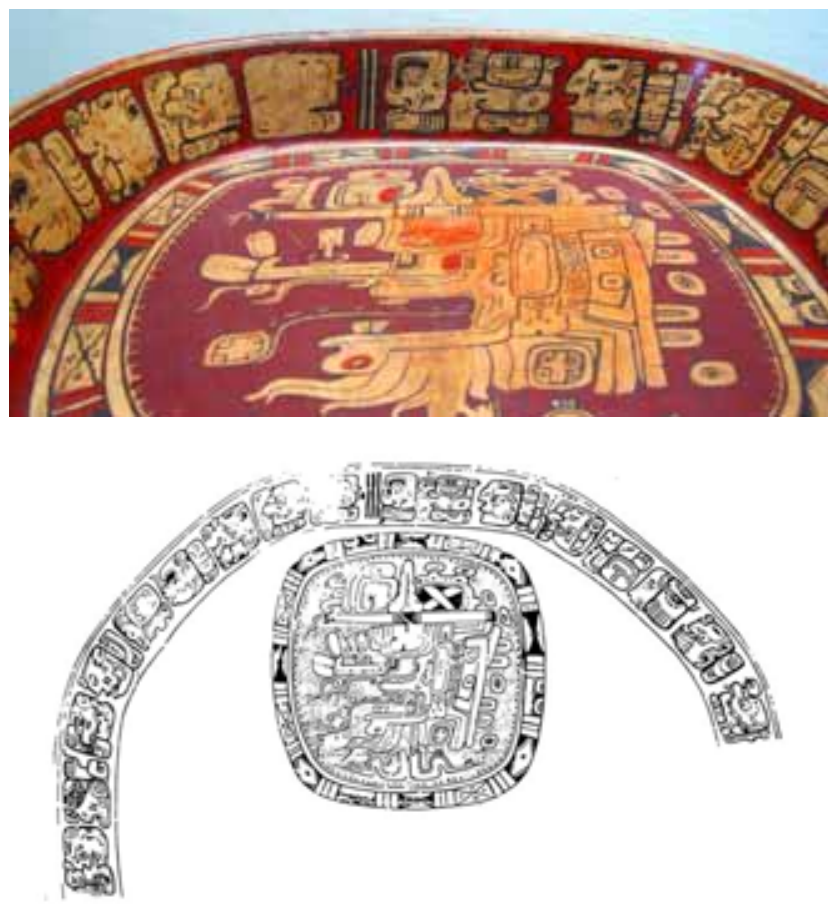

Figura 4. Nombre del gobernante de 'IK' TAYal Chan K'inich I (ta-YAl-CHAN-na K'INICH), escrito en la Fórmula Dedicatoria de la vasija 1 del entierro 30 de Dos Pilas. Dibujo de Stephen D. Houston.

Tayal / Tayel Chan K'inich I

Todo esto muestra que algunos de los señores de 'Ik' gozaban de cierta libertad de acción, la cual podría verse reflejada en un hallazgo sorprendente: la vasija 1 del entierro 30 de Dos Pilas (figura 4), cuya cripta fue encontrada dentro de la Estructura L5-1 por el equipo de Arthur Demarest. La sepultura parece datar de octubre de 726, según la Estela 8 de Dos Pilas (I7-I16a), ubicada frente a la escalinata del edificio (ver Demarest, 1993: 100, 104; Demarest et al., 1992; 
Houston, 1992; 1993: 110; Martin y Grube, 2008: 59), mientras que la vasija 1 es un plato tetrápode ( $k^{\prime} a h n$ 'ookwante') ${ }^{12}$ en cuya Fórmula Dedicatoria se encuentra el nombre de un gobernante de 'Ik' denominado Tayal Chan K'inich. ${ }^{13}$ Su reino tal vez tuvo lugar durante el primer tercio del siglo vill. Aunque se desconoce la identidad del individuo sepultado en el entierro 30, lo más probable es que se trate de 'Itzamnaaj K'awiil (698-726 d.C.), ${ }^{14}$ mencionado en la misma Estela 8 de Dos Pilas, que contiene la fecha de su muerte y datos detallados sobre su sepelio. Extrañamente, la mención de su fallecimiento también se encuentra en uno de los huesos labrados (MT-28) de la tumba de Jasaw Chan K'awiil (ver Trik, 1963: 17), su adversario de Tikal. Según Houston (1993: 111), ello puede indicar la existencia de un parentesco entre ambos soberanos, pues el linaje de Dos Pilas era una rama escindida del de Tikal. Desconocemos si los señores de Motul tuvieron algún parentesco con los de Tikal, pero es posible que hayan existido lazos consanguíneos entre las dos casas dinásticas, y que esto explique la presencia del plato tetrápode en el entierro 30 de Dos Pilas. Otra posibilidad es simplemente que algunos señores de 'Ik', aprovechando su relativa autonomía, hayan tratado de establecer pactos de no agresión con los peligrosos señores de Dos Pilas, en cuyo intento organizaron banquetes para funcionarios del Petexbatun, obsequiando vasijas decoradas o mandándolas en alguna visita o intercambio de presentes (Demarest et al., 1992: 293). ${ }^{15}$ Finalmente, también se ha especulado que este plato llegó a Dos Pilas como botín de guerra, idea que encuentra cierto fundamento en un fragmento de panel hallado en 1991 por Robert Chatham, mismo que parece documentar la existencia de una relación hostil entre los señores de 'Ik' y de Mutu'l (Dos Pilas) (Houston, 1992: 277).

Es preciso mencionar que Tayel Chan K'inich ya era conocido por al menos dos vasijas sin contexto arqueológico: K2573 (figura 5a) y K4996. Tal como observan Thomás G. Garrison y David S. Stuart (2004: 833), la Fórmula Dedicatoria de este último vaso sugiere que perteneció a una mujer de Xultun ('IX-? wi-tzi'AJAW); su imagen representa a Tayel Chan K'inich recibiendo el tributo (patan) de mano de sus tres lakam (ver Houston y Stuart, 2001:69; Lacadena, 2006: 2-3)

\footnotetext{
${ }^{12}$ Este nombre tipológico, que incluye el de los sustantivos 'banco' o 'silla' (k'ahn) y 'pie, pata' o 'soporte' ('ook), más la terminación -wante', de platos hondos, fue identificado por Alfonso Lacadena (comunicación personal, 19 de marzo de 2007).

${ }^{13} \mathrm{El}$ nombre de este gobernante es difícil de leer. Una parte del mismo quizá podría descifrarse como Chaahk 'Ub'ook(?), Waklaju'n 'Ook'in(?) Tayal Chan K'inich. La lectura tentativa del logograma T533 como B'OK, b'ook, 'aroma, fragancia, olor' o 'perfume' fue sugerida por Christian Prager (2006 con base en una colocación atestiguada en la página 36a del Códice de Madrid (b'o-T533-ki). Finalmente, debemos a Boot (comunicación personal, 27 de noviembre de 2007) la lectura del cartucho T53.561c:670:23 como ta-YAL?-CHAN-na, Tayal(?) Chan.

${ }^{14}$ La lectura actualizada del nombre de este mandatario tal vez deba ser Kokaaj(?) K'awiil (KOKAJ?-K'AWIL).

${ }^{15}$ Este entorno de inestabilidad política y habilidad diplomática pudo ser el motor que impulsó la elaboración de las vasijas de la tradición 'Ik', como claramente explican Matthew G. Looper, Dorie J. Reents-Budet y Ronald L. Bishop (2009: 148-150).
} 


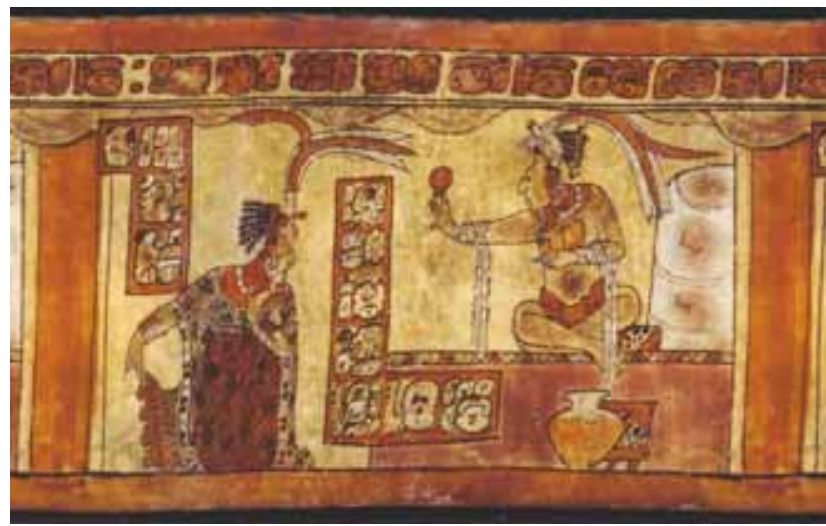

(a)

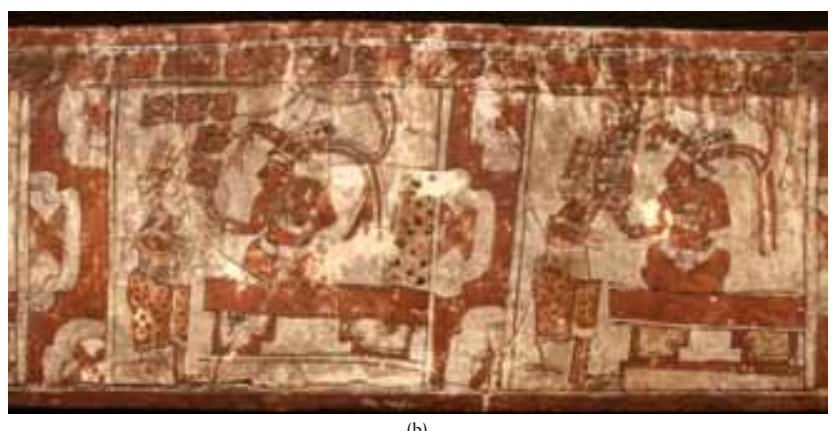

(b)

FigurA 5. (a) Fotografía desplegada del vaso K2573 con un pasaje de la vida de Tayel Chan K'inich I. (b) Fotografía desplegada del vaso K7997 del entierro 116 de Tikal, que es la tumba de Jasaw Chan K'awiil 1.

Fotografías de Justin Kerr. 
en la fecha 9.15.3.6.6 (6 de diciembre de 734 d.C.). Por su parte, el vaso K2573 (figura 5a) contiene un ejemplo más detallado de su nombre, Tayel Nichan K'inich Nich'ich'(?) (ta-ye-le ni-CHAN-na-K'INICH ni-CH'ICH'?), que concluye con el glifo emblema de 'Ik'. En la escena parece estar recibiendo a una elegante mujer que aparentemente procede de un lugar denominado ko-"ALTAR.DE.PIEDRA" y porta el título de 'Ix Mutu'l 'Ajaw, 'señora de Tikal. ${ }^{16}$ De acuerdo con Houston (2006: 3), el topónimo ko-"ALTAR.DE.PIEDRA" designa a una localidad de ubicación desconocida, aunque presumiblemente se encontraba entre Naranjo y Caracol. La vasija K2573 guarda fuertes consonancias estilísticas con un grupo de vasos del complejo Imix (700-850 d.C.) encontrados en el entierro 116 del Templo I de Tikal (figura 5b), ni más ni menos que la tumba de Jasaw Chan K'awiil I (ver Trik, 1963; Foncerrada de Molina y Lombardo de Ruiz, 1979: 234-251; Culbert, 1993: figuras 69-75). ${ }^{17}$ Entre las semejanzas se encuentran el uso de bandas rojas anchas que enmarcan los bordes superior e inferior de los vasos, algunas veces auxiliados por líneas paralelas negras que delimitan las bandas o los textos de la Fórmula Dedicatoria, una paleta definida que incluye rojo, anaranjado, blanco y negro sobre un engobe de color crema, así como la temática abordada y composición pictórica empleada: escenas de encuentro con un dignatario sentado sobre su banqueta o trono, en el interior de una habitación con cortinas y entrepaños que pueden enmarcarse por bandas verticales delgadas del mismo color que el fondo. Aunque se dintinguen diferentes manos en los vasos del entierro 116 , parece indudable que éstos y el K2573 (figura 5a) corresponden a una misma época e impulso artístico. ${ }^{18}$ Ello confirmaría que Tayel Chan K'inich efectivamente gobernó hacia 734 d.C., puesto que Yik'iniiy Chan K'awiil (Gobernante B) se entronizó en Tikal el 12 de diciembre de ese mismo año ${ }^{19}$ y un poco antes debió haberse cerrado la tumba de su predecesor (Martin y Grube, 2008:47).

${ }^{16}$ Coe y Kerr (1997: 17), Schele y Freidel (1990) y MacLeod y Kerr (1994: 84) consideraron que a mujer procedía de Dos Pilas, dado que esta ciudad comparte con Tikal el mismo glifo emblema (Mutu'l). No obstante, me parece más lógico que ella proceda de Tikal, en virtud de que el topónimo ko-"ALTAR.DE.PIEDRA" que porta en sus glifos nominales designa alguna localidad del Petén orienta (ver Houston, 2006: 2-3). Dmitri Beliaev (comunicación personal, 5 de diciembre de 2007) sugiere que puesto que el signo principal de su glifo emblema - tal como aparece en el vaso K2573 (figura 5a) - carece del nudo propio de los emblemas de Dos Pilas y Tikal, esta mujer podría ser una seño5a) - carece del nudo propio de los emblemas de Dos Pilas y Tikal, esta mujer podría ser una seño ra de Yomootz, lugar ubicado en el área de Xultun. Podemos especular también que esta dama se presenta ante Tayel Chan Kinich para convertirse en su esposa, dado que en las inscripciones un expresión que delata la presencia de consortes extranjeras es huli, 'ella arribó' (ver Schele y Grube, 1994: 110; Houston y Stuart, 2001: 77, nota 4; Freidel y Guenter, 2003: 2).

${ }^{17}$ En la nomenclatura de Kerr esos vasos son: K7996, K7997 (figura 5b), K7998, K7999, K8000, K8001, K8002 у K8003.

${ }^{18} \mathrm{~A}$ este mismo impulso estético pudo responder el interesante vaso K7821, que fue estudiado por Marc U. Zender (2005: 10-11).

${ }^{19}$ Schele y Freidel (1990: 478, nota 13) apuntan que la madre de Yik'iniiy Chan K'awiil, la señora Lachan 'Une' Mo', procedía de la entidad política de Maan, según se atestigua en la Estela 5 de Tikal: D7-D9 (véase Jones y Satterthwaite, 1983: figura 8). Ello cerraría el círculo de relaciones diplomáticas entre los señoríos de 'Ik' (Motul de San José), Maan (La Florida) y Mutu'l (Tikal). 
Uno de los desechos de manufactura encontrados en el basurero ubicado al noroeste de la Acrópolis de Motul de San José, se caracteriza por la misma paleta roja y anaranjada sobre fondo crema, semejantes patrones de composición y misma temática cortesana; Foias (2003a: 22) misma reconoce que el estilo de este tiesto se parece al de Tikal, aunque la finura de sus dedos es característica del corpus 'Ik'. Tal vez este fragmento date de la época de Tayel Chan K'inich I.

\section{Yajawte' K'inich}

La fecha de ascensión del siguiente soberano de 'Ik' es incierta todavía. El vaso K2795 nos presenta al llamado Cacique Gordo bajando de una litera o trono portátil (véase Reents-Budet, 2001: 196), aparentemente para presenciar el sacrificio de un cautivo sobre un tablado de madera (ver Schele y Miller, 1986: 227-228; Taube, 1988:340-350; 1994:671-674). El andamio, que se encuentra provisto de techumbre y escalera de mano, recuerda las estructuras labradas en algunas estelas de Piedras Negras, donde la ceremonia de entronización de los reyes incluye la inmolación de una víctima (ver Proskouriakoff, 1960:455; Stuart, 1988:195; Velásquez García, 2006: 4; Looper, Reents-Budet y Bishop, 2009: 144). Aunque el día del tzolk'iin está casi borrado, es posible que sea Oc, lo que daría una fecha de 9.15.9.4.10, 30 de septiembre de 740 (Reents-Budet et al., 2007:1153, tabla 2). Un detalle importante es que a la ceremonia de entronización del Cacique Gordo asistió su sucesor en el mando, que llevaba por nombre K'inich Lamaw 'Ek', información que se desprende de la vasija K1463 (ver Looper, Reents-Budet y Bishop, 2009: 142-143).

$\mathrm{Al}$ menos tres de los vasos del Cacique Gordo llevan la firma de un pintor llamado Tub'al 'Ajaw o 'Señor de Tub'al', lugar ubicado en algún sitio entre Tikal y Naranjo. ${ }^{20}$ Tub'al 'Ajaw y otros artistas anónimos representaron a su señor en diversos vasos con escenas de danza, acompañadas por autosacrificio y disfraces fantásticos. Su nombre aparece escrito en al menos diez vasijas pintadas (ReentsBudet et al., 2007:1143), ${ }^{21}$ lo que permite leerlo como Yajawte' K'inich. Cabe

${ }^{20}$ Guenter (comunicación personal, 9 de noviembre de 2007) opina que el sitio de Tub’al puede identificarse con Nakum o estar cerca de Nakum, pues de acuerdo con el Dintel 2 del Templo IV de Tikal (B3-B6), Yik'iniiy Chan K'awiil (734-746 d.C.) salió de su ciudad y llegó a Tub'al un día antes del ataque contra Naranjo, y un ejército sólo puede recorrer en un día la mitad de la distancia que existe entre Tikal y Naranjo (ca. $40 \mathrm{~km}$ ). Justo en medio de las dos ciudades se encuentra Nakum. No obstante, el glifo emblema atestiguado en las inscripciones de Nakum no parece identificarse con Tub’al (Grube, comunicación personal, 15 de noviembre de 2007), y ese topónimo debe encontrarse en alguna otra parte de la misma región. Zender (2005: 14) opina simplemente que Tub'al pudo haberse localizado en la vecindad de Naranjo, mientras que Tokovinine (2006b: 370) coincide en que se trata de un sitio de la región de los lagos, ubicado más o menos a la misma distancia de Naranjo y de Tikal.

${ }^{21}$ K533 (ya-'AJAW-TE'?-K'INICH), K534 (YAJAW?-TE' K'INICH), K791 (ya-'AJAW-TE' K'INICH), K1399 (YAJAW?-TE' K'INICH), K1439 (ya-'AJAW-TE' K'INICH), K1452 (ya-'AJAW-TE' K'INICH), K1463 (ya-|"'AJAW-'TE'] K'INICH), K1896 (ya-'AJAW-TE' K'INICH), K3054 (["ya]-'AJAW-['TE'] K'INICH) y K4120 (YAJAW?-TE'-K'INICH).

VELASQUEZ / LOS SEÑORES DE LA ENTIDAD POLÍtICA DE 'IK' 
mencionar que este soberano incorporó a sus cartuchos nominales la expresión 'uchanul 'lk' B'ul, 'guardián de 'Ik' B'ul', probable alusión a un cautivo de guerra prominente llamado 'Ik' B'ul (Prager, 2008: 4).

Ya como gobernante emprendió una danza autosacrificial con sonajas de calabaza, armazón redondo sobre la espalda y largas plumas negras, que está representada en el vaso K1399 y tuvo lugar posiblemente en noviembre de 744 (9.15.13.7.19). En mayo de 745 (9.15.13.17.10), probablemente, ejecutó un baile sagrado donde personificó al dios solar nocturno Huk Chapaht Tz'ikiin K'inich. Exactamente cuatro años después, en mayo de 749 (9.15.18.0.10), parece haber emprendido una extraña danza llamada ti t'olo[l] b'ahlam, ${ }^{22}$ 'con el jaguar [en] ringlera' (figura 6), donde se apoya en el lomo de un contorsionista vestido como jaguar del inframundo, mientras que un siervo arrodillado le sostiene la canasta con tiras de papel ensangrentado (Grube, 1992: 215). El estilo de los vasos donde se encuentran pintadas estas danzas, así como su misma temática y atuendos, guardan fuertes semejanzas con un vaso de la tradición 'Ik' que fue encontrado en Ixkun (ver Tokovinine, 2006b: 367-372).

Yajawte' K'inich parece haber tenido una especial predilección por el recurso plástico de la máscara de "rayos X" (véase Velásquez García, 2007), misma que se encuentra en al menos diez vasos de su época, ${ }^{23}$ así como en el lado oeste de la Estela 2 de Motul de San José.

El danzante grabado en el lado derecho de la cara oriente de dicha estela (Maler, 1910: plate 45) es probablemente Yajawte' K'inich (figura 7). Ello se aprecia no sólo en el aspecto semi obeso del personaje, sino en el formato mismo de la composición, con ambos danzantes representados frontalmente, pero sus rostros de perfil, orientados al eje central o locus de la escena. Como ha mostrado Houston (1998: 342), el lado derecho de las composiciones era el más favorecido dentro de las convenciones artísticas mayas, y en dicha posición parece encontrarse el gobernante de 'Ik'. Tatiana A. Proskouriakoff (1950: 142, 191; 1999: 151.) fechó esta estela por estilo hacia 770 d.C., con un margen de \pm dos k'atunes, lo que concuerda con la época estimada para el régimen de Yajawte' K'inich ( $c a$. $740-755 / 756$ d.C.). Este se hace acompañar de otro danzante cuya estatura y volumen corporal son ligeramente menores que los del soberano. Su posición, a la izquierda, sugiere que se trata de un visitante o de un noble de menor rango

${ }^{22}$ Agradezco a Albert Davletshin por haber discutido conmigo algunas ideas sobre dicho pasaje (1 de marzo de 2007).

${ }^{23}$ K533, K1439, K1896, K2025, K2795, K3054, K4606, K6888 y K5418, así como el vaso A 886 de Ixkun (Tokovinine, 2006b: 367-372). Christina T. Halperin (2006: 32) ha notado que entre las figurillas de terracota encontradas en Motul de San José hay varias con máscaras desmontables que fueron hechas por separado. En su opinión, éstas pueden representar seres humanos enmascarados, un tema paralelo al de los danzantes representados en los vasos 'lk'. Por otra parte, el análisis de activación de neutrones practicado sobre estas figurillas revela que corresponden a los mismos grupos químicos (y probablemente a los mismos talleres) de las vasijas ' Ik' y de algunas piezas policromas encontradas en Motul de San José (Halperin, 2006: 12, 47; Reents-Budet et al., 2007:1145). 


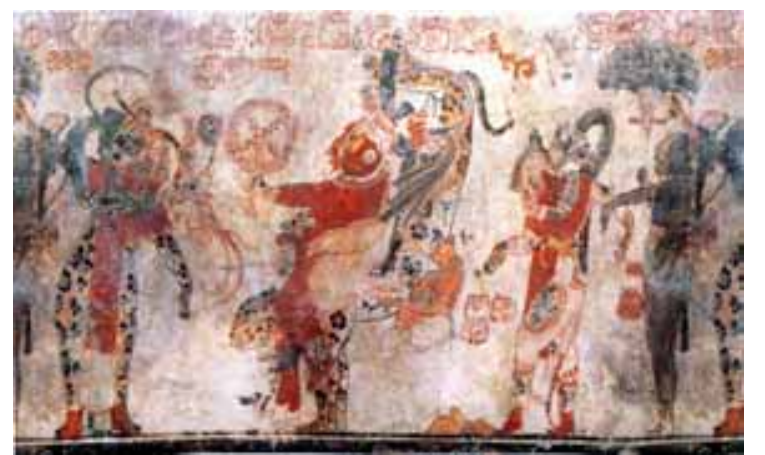

Figura 6. Fotografí desplEGADA del vaso K1439 o MS1121, del Museum of Fine Arts de Boston. Atribuido al pintor Tub'al 'Ajaw. Representa a Yajawte' K'inich y otros miembros de su corte involucrados en una danza con un jaguar contorsionista (t'olol b'ahlam). Fotografía de Justin Kerr (tomada de Reents-Budet et al., 1994:166).

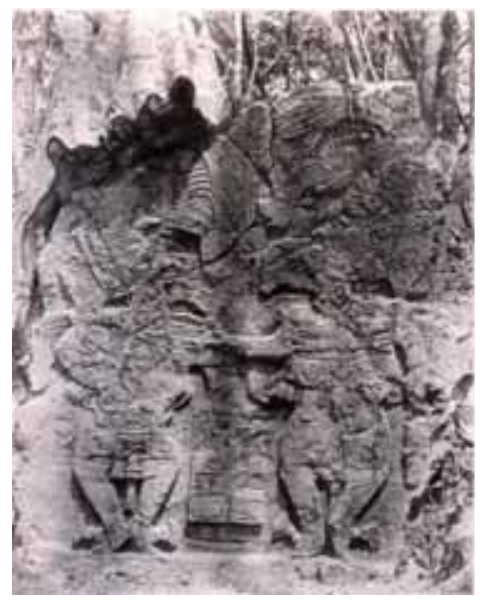

Figura 7. Lado este (posterior) de la Estela 2 de Motul de San José, ubicada en la Plaza B del Grupo B. Representa a Yajawte' K'inich, señor de 'lk', ejecutando un baile en compañía de Ju'n Tzahk To'k', señor de 'Itza'. Fotografiada por Teobert Maler (1910: 171). 
(Houston, 1998: 342; Houston y Stuart, 2001: 62-63.). Su nombre se encuentra escrito como Ju'n Tzahk To'k' e incluye el título de señor divino de 'Itza', linaje que pudo haber residido, de acuerdo con Erik Boot, ${ }^{24}$ en la cercana ciudad de Itzimté (véase Mejía, 2003).

Durante el gobierno de Yajawte' K'inich la entidad política de 'Ik' parece haber entrado en conflicto con Dos Pilas. Por una parte, el gobernante que erigió la Estela 11 de Machaquilá (Graham, 1967: 84, figura 63), en junio de 741 (9.15.10.0.0), se declara como el 'supervisor' de un señor de 'Ik' (figura 8a). Dos años más tarde, K'awiil Chan K’inich (741-761 d.C.) de Dos Pilas inició una campaña bélica contra sus enemigos asentados al norte del río de la Pasión, entre los cuales se encontraban El Chorro (743 d.C.), Yaxchilán (745 d.C.) y Motul de San José (745 d.C.) (Houston, 1993: 117, 124; Martin y Grube, 2002: 62). Sus victorias se encuentran consignadas en los escalones 1 y 2 de la Escalera Jeroglífica 3 de Dos Pilas. El prisionero de Motul recibe el nombre de Chuliw Hix (figura $8 \mathrm{~b}$ ) y fue capturado el 25 de abril de 745 (9.15.13.15.19, 11 Cauac 2 Tzec), tres días después que el de Yaxchilán, lo que sugiere que ya entonces los linajes de 'Ik' (Motul de San José) y de Pa'chan (Yaxchilán) pudieron estar asociados en acciones de guerra.

Dos Pilas y Motul de San José parecen haber intercambiado algunos elementos culturales, como lo testifica la presencia de tiestos de cerámica 'lk' que han sido hallados en la propia Dos Pilas (Reents-Budet et al., 2007:1144). ${ }^{25}$ Esto ha hecho suponer a Foias (1999: 948; 2003a: 19; 2003b: 12) que a raíz de la captura de Chuliw Hix, en 745, la relación entre Tikal y Motul de San José se rompió y la entidad política de 'Ik' pasó a la hegemonía de Dos Pilas. No obstante, aunque el rango político de los cautivos de K'awiil Chan K'inich era ciertamente elevado, ya que eran nobles o 'ajawtaak, no se trataba de gobernantes o señores divinos, y al menos en el caso de Motul de San José no existen pruebas de que la captura de Chuliw Hix haya afectado la sucesión dinástica local. ${ }^{26}$

En 1961, Richard E. W. Adams (1963; 1971:68-78; Stuart, 1975: 774-776; Foncerrada de Molina y Lombardo de Ruiz, 1979:35; Schele, 1988: 295-299) encontró el famoso vaso K3120 en el entierro 96 de Altar de Sacrificios (figura 9a). La crip-

${ }^{24}$ Correspondencia enviada el 16 de septiembre de 2006. También ver la discusión histórica sobre el título 'Itza', en el excelente libro de Erik Boot (2005: 23-193, 453-456).

${ }^{25}$ Joel Palka, comunicación personal, 12 de marzo de 2007. Afinidades estilísticas, temáticas y epigráficas pueden encontrarse también entre los vasos del corpus 'lk' y algunas vasijas que proceden del Petexbatun (Reents-Budet et al., 1994: 225-226, nota 30). Lo mismo puede decirse de la difusión del recurso iconográfico de la máscara de "rayos X", que podemos también encontrar en las estelas sinópticas 2 de Aguateca y 16 de Dos Pilas (Velásquez García, 2007), así como de ciertos rasgos del patrón de asentamiento entre Motul de San José, Trinidad de Nosotros y la propia Dos Pilas (Moriarty, 2004b: 31).

${ }^{26}$ Aunque se ha especulado que la captura del señor de Yaxchilán (en 745 d.C.) pudo haber ocasionado el interregno de diez años que tuvo lugar luego de la muerte de 'Itzamnaaj B'ahlam II (681-742 d.C.) (Houston, 1993: 117), lo cierto es que ese vacío de poder había comenzado casi tres años antes de la batalla. 


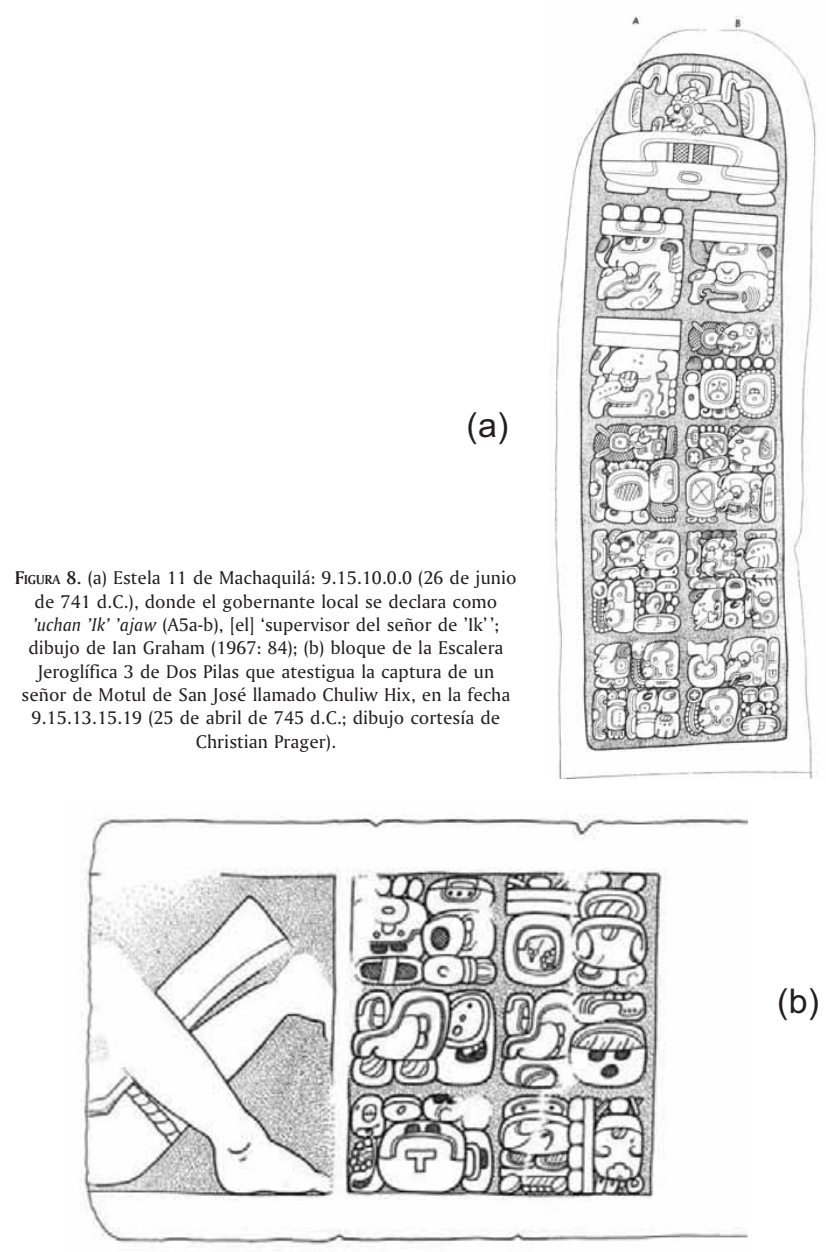

VELASQUEZ / LOS SEÑORES DE LA ENTIDAD POLÍtica DE 'IK' 
ta funeraria contenía el esqueleto de una mujer joven, mientras que el recipiente fue consagrado en abril de $754(9.16 .3 .0 .0)$ y es obra de otro pintor cuyo nombre está parcialmente descifrado: Mo...n B'uluch Laj. Su estilo inconfundible, con seres sobrenaturales que flotan en un ambiente onírico (véase Quirarte, 1979; Grube y Nahm, 1994), fue reproducido también en el vaso K791 (figura 9b), activado ritualmente en enero de $755(9.16 .3 .13 .14) .{ }^{27}$ Cabe mencionar que aunque estas vasijas se asocian de algún modo con Yajawte' K'inich, Mo...n B'uluch Laj parece haber trabajado también para el siguiente gobernante de 'Ik' (Reents-Budet et al., 2007: 1143).

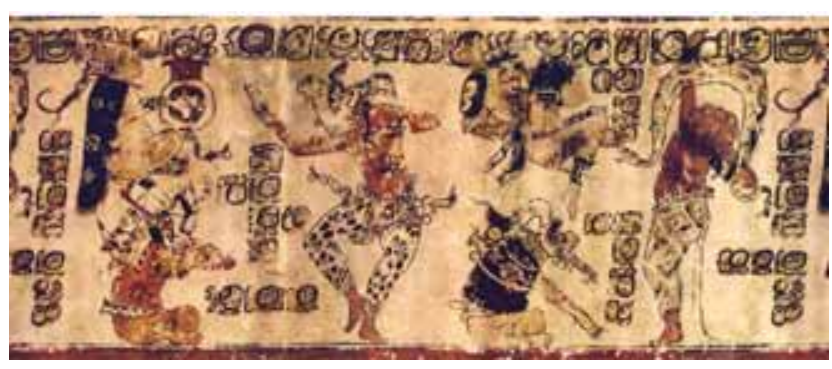

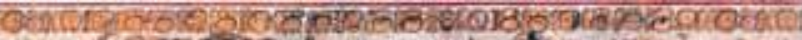

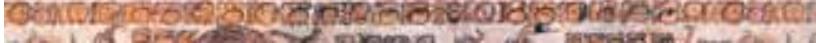

